\title{
Attacks on the Birational Permutation Signature Schemes
}

\author{
Don Coppersmith \\ IBM Research \\ T. J. Watson Research Center \\ Yorktown Heights, NY 10598
}

\author{
Jacques Stern, Serge Vaudenay \\ Laboratoire d'Informatique \\ Ecole Normale Supérieure \\ 75230 Paris, France
}

\begin{abstract}
Shamir presents in [3] a family of cryptographic signature schemes based on birational permutations of the integers modulo a large integer $N$ of unknown factorization. These schemes are attractive because of the low computational requirements, both for signature generation and signature verification. However, the two schemes presented in Shamir's paper are weak. We show here how to break the first scheme, by first reducing it algebraically to the earlier Ong-Schnorr-Shamir signature scheme, and then applying the Pollard solution to that scheme. We then show some attacks on the second scheme. These attacks give ideas which can be applied to schemes in this general family.
\end{abstract}

\section{The first scheme}

The public information in Shamir's first scheme consists of a large integer $N$ of unknown factorization (even the legitimate users need not know its factorization), and the coefficients of $k-1$ quadratic forms $f_{2}, \cdots, f_{k}$ in $k$ variables $x_{1}, \cdots, x_{k}$ each. Each of these quadratic forms can be written as

$$
f_{i}=\sum_{j, \ell} \alpha_{i j \ell} x_{j} x_{\ell}
$$

where $i$ ranges from 2 to $k$ and the matrix $\alpha_{i j \ell}$ is symmetric i.e. $\alpha_{i j \ell}=\alpha_{i \ell j}$.

The secret information is a pair of linear transformations. One linear transformation $B$ relates the quadratic forms $f_{2}, \cdots, f_{k}$ to another sequence of quadratic forms $g_{2}, \cdots, g_{k}$. The second linear transformation $A$ is a change of coordinates that relates the variables $\left(x_{1}, \cdots, x_{k}\right)$ to a set of "original" variables $\left(y_{1}, \cdots, y_{k}\right)$. Denoting by $Y$ the column vector of the original variables and by $X$ the column vector of the new variables, we can simply write $Y=A X$.

Of course, the coefficients of $A$ and $B$ are known only to the legitimate user. The trap-door requirements are twofold: when expressed in terms of the original variables $y_{1}, \cdots, y_{k}$, the quadratic form $g_{2}$ is computed as:

$$
g_{2}=y_{1} y_{2}
$$

and the subsequent $g_{i}$ 's, $3 \leq i \leq k$ are sequentially linearized, i.e. can be written

$$
g_{i}\left(y_{1}, \cdots, y_{k}\right)=l_{i}\left(y_{1}, \cdots, y_{i-1}\right) \times y_{i}+q_{i}\left(y_{1}, \cdots, y_{i-1}\right)
$$


where $l_{i}$ is a linear function of its inputs and $q_{i}$ a quadratic form.

To sign a message $M$, one hashes $M$ to a $k-1$-tuple $\left(f_{2}, \cdots, f_{k}\right)$ of integers modulo $N$, then finds a sequence $\left(x_{1}, \cdots, x_{k}\right)$ of integers modulo $N$ satisfying (1). This is easy from the trap-door.

We let $A_{i}, 2 \leq i \leq k$ denote the $k \times k$ symmetric matrix of the quadratic form $f_{i}$, namely:

$$
A_{i}=\left(\alpha_{i j \ell}\right)_{1 \leq j \leq k, 1 \leq \ell \leq k}
$$

The kernel $K_{i}$ of $g_{i}$ is the kernel of the linear mapping whose matrix is $A_{i}$. It consists of vectors which are orthogonal to all vectors with respect to $g_{i}$. The rank of the quadratic form $g_{i}$ is the rank of $A_{i}$. It is the dimension of $K_{i}$ as well as the unique integer $r$ such that $g_{i}$ can be written as a sum of squares of $r$ independent linear functionals. Actually, all this is not completely accurate as $N$ is not a prime number and therefore $\mathbf{Z} / N$ is not a field. This question is addressed at the end the paper and, meanwhile, we ignore the problem.

An easy computation shows that $K_{i}$ is the subspace defined in terms of the original variables by the equations

$$
y_{1}=\cdots=y_{i}=0
$$

It follows from this that

i) $K_{i}$ is decreasing

ii) the dimension of $K_{i}$ is $k-i$

iii) any element of $K_{i-1}$ not in $K_{i}$ is an isotropic element wrt $g_{i}$, which means that the value of $g_{i}$ is zero at this element.

We will construct a basis $b_{i}$ of the $k$-dimensional space, such that $b_{i+1}, \cdots, b_{k}$ spans $K_{i}$ for $i=2, \cdots, k-1$. The main problem we face is the fact that the $g_{i}$ 's and therefore the $K_{i}$ 's are unknown. In place, we know the $f_{i}$ 's. We concentrate on the (unknown) coefficient $\delta_{i}$ of $g_{k}$ in the expression of $f_{i}$, i.e. we write

$$
f_{i}=\delta_{i} g_{k}+\sum_{j=2}^{k-1} \beta_{i j} g_{j}
$$

As coefficients have been chosen randomly, we may assume that $\delta_{k}$ is not zero. Let $i<k$. Consider the quadratic form $Q_{i}(\lambda)=f_{i}-\lambda f_{k}$. When $\lambda=\delta_{i} / \delta_{k}$, this form has a non-trivial kernel and therefore $\delta_{i} / \delta_{k}$ is a root of the polynomial $P_{i}(\lambda)=$ $\operatorname{det}\left(Q_{i}(\lambda)\right)$. This is not enough to recover the correct value of $\lambda$. Computing the matrix of $Q_{i}(\lambda)$ for $\lambda_{i}=\delta_{i} / \delta_{k}$ in the basis corresponding to the original coordinates $y_{1}, \cdots, y_{k}$ yields the following

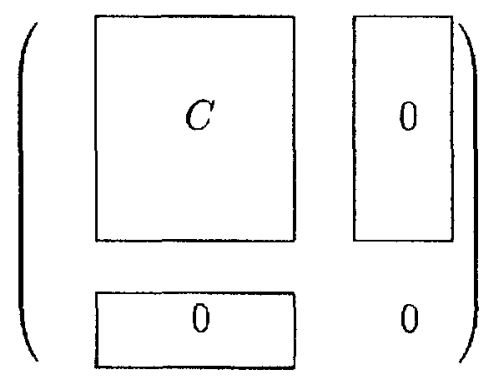


In the same basis, the matrix of $Q_{i}(\lambda)$ for any $\lambda$, can be written as

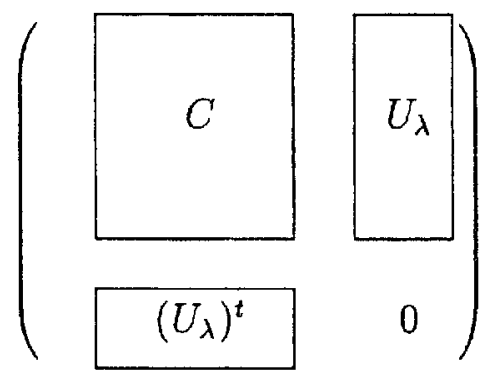

We observe that $U_{\lambda}$ is linear in $\lambda$ and vanishes at $\lambda_{i}$. Since determinants canl be computed up to a multiplicative constant in any basis, it follows that $\left(\lambda-\lambda_{i}\right)^{2}$ factors out in $P_{i}(\lambda)$. Thus the correct, value of $\lambda_{i}$ can be found by observing that it is a double root of the polynomial equation $P_{i}(\lambda)=0$. This double root is disclosed by taking the g.c.d. $(\bmod N)$ of $P_{i}$ and $P_{i}^{\prime}$ with respect to $\lambda$. We find a linear equation in $\lambda$, from which we easily compute $\lambda_{i}$.

Once all coefficients $\lambda_{i}$ have been recovered, we set for $i=2, \cdots, k-1$

$$
\tilde{f}_{i}=f_{i}-\lambda_{i} f_{k} \quad i<k
$$

and $\tilde{f}_{k}=f_{k}$. We note that all quadratic forms $\tilde{f}_{i}$ have kernel $K_{k-1}$. This allows to pick a non-zero vector $b_{k}$ in $K_{k-1}$. The construction can then go on inductively in the quotient space of the $k$-dimensional space by the vector spanned by $\left\{b_{k}\right\}$ with $\tilde{f}_{2}, \cdots, \tilde{f}_{k-1}$ in place of $f_{2}, \cdots, f_{k}$.

At the end of the recursive construction, we obtain a sequence $b_{i}, 3 \leq i \leq k$ such that $b_{i+1}, \cdots, b_{k}$ spans $K_{i}$ for $i=2, \cdots, k-1$ and a sequence of quadratic forms $\tilde{f}_{2}, \cdots, \tilde{f}_{k}$ such that

i) $\tilde{f}_{i}$ has kernel $K_{i}$

ii) $b_{i}$ is an isotropic element wat $\tilde{f}_{i}$

Choosing $b_{1}, b_{2}$ at random, we get another set of coordinates $z_{1}, \cdots, z_{k}$ such that i) $\tilde{f}_{2}$ is a quadratic form in the coordinates $z_{1}, z_{2}$

ii) $\tilde{f}_{3}, \cdots, \tilde{f}_{k}$ is sequentially linearized

The rest is easy. From a sequence of prescribed values for $f_{2}, \cdots, f_{k}$, we can compute the corresponding values of $\tilde{f}_{2}, \cdots, \tilde{f}_{k}$. Next, we can find values of $\left\{z_{1}, z_{2}\right\}$ achieving a given value of $\tilde{f}_{2}(\bmod N)$ in exactly the same way as the Pollard solution of the Ong-Schnorr-Shamir scheme [2]. Then, values for $z_{3}, \cdots, z_{k}$ achieving given values of $\tilde{f}_{3}, \cdots, \tilde{f}_{k}$ are found by successively solving $k-2$ linear equations. Finally, the values of $z_{1}, \cdots, z_{k}$ can be translated into values of $x_{1}, \cdots, x_{k}$.

Example. In Shamir's paper [3], an example is given with $N=101$. (We use 101 to maintain consistency with Shamir's paper, even though 101 is prime, while $N$ should be composite. We treat 101 as a number of unknown factorization; in particular we never solve nonlinear equations $\bmod 101$.)

$$
v_{2}=78 x_{1}^{2}+37 x_{2}^{2}+6 x_{3}^{2}+54 x_{1} x_{2}+19 x_{1} x_{3}+11 x_{2} x_{3} \quad(\bmod 101)
$$




$$
v_{3}=84 x_{1}^{2}+71 x_{2}^{2}+48 x_{3}^{2}+44 x_{1} x_{2}+33 x_{1} x_{3}+83 x_{2} x_{3} \quad(\bmod 101)
$$

Matrices of $f_{2}, f_{3}$ are as follows

$$
\left(\begin{array}{ccc}
78 & 2760 \\
27 & 37 & 56 \\
60 & 56 & 6
\end{array}\right) \quad\left(\begin{array}{l}
842267 \\
227192 \\
679248
\end{array}\right)
$$

We get:

$$
\begin{gathered}
P(\lambda)=\operatorname{det}\left(f_{2}-\lambda f_{3}\right)=34\left(\lambda^{3}+75 \lambda^{2}+55 \lambda+71\right) \\
P^{\prime}(\lambda)=\lambda^{2}+50 \lambda+52 \\
\operatorname{gcd}\left(P, P^{\prime}\right)=\lambda-63
\end{gathered}
$$

We let

$$
\tilde{f}_{2}=f_{2}-63 f_{3} \quad ; \quad \tilde{f}_{3}=f_{3}
$$

The kernel of $\tilde{f}_{2}$ is spanned by vector $b_{3}=(31,12,1)^{t}$. We pick $b_{2}=(0,1,0)^{t}$ and $b_{1}=(1,31,0)^{t}$. We get, in the corresponding coordinates $z_{1}, z_{2}, z_{3}$ :

$$
\tilde{f}_{2}=26 z_{1}^{2}+8 z_{2}^{2} \quad ; \quad \tilde{f}_{3}=z_{3}\left(26 z_{1}+20 z_{2}\right)+90 z_{1}^{2}+2 z_{1} z_{2}+71 z_{2}^{2}
$$

\section{The second scheme}

We now treat Shamir's [3] second scheme. The ideas developed in this section will have general applicability.

Throughout, we will pretend we are working in $\mathrm{Z} / p$ rather than $\mathbf{Z} / N$.

We treat first the case $s=1$. We begin with $k$ variables $y_{1}, y_{2}, \ldots, y_{k}$, with $k$ odd. These are subjected to a secret linear change of variables which gives $u_{i}=\sum_{j} a_{i j} y_{j}, i=1,2, \ldots, k$, with the matrix $A=\left(a_{i j}\right)$ secret. The products $u_{i} u_{i+1}$, including $u_{k} u_{1}$, are subjected to a second secret linear transformation $B=\left(b_{i j}\right)$, so that $v_{i}=\sum_{j} b_{i j} u_{j} u_{j+1}, i=1,2, \ldots, k-1$. The public key is the set of coefficients $\left(c_{i j \ell}\right)$ expressing $v_{i}$ in terms of pairwise products $y_{j} y_{\ell}$, for $1 \leq i \leq k-1$,

$$
v_{i}=\sum_{j, \ell} c_{i j \ell} y_{j} y_{\ell}, 1 \leq i \leq k-1, c_{i j \ell}=c_{i \ell j}
$$

(Here $i$ is ranging to $k-1$, so we have discarded $s=1$ of the $v_{i}$.)

The first step in our solution: linear combinations of the $v_{i}$ are linear combinations of the $u_{i} u_{i+1}$, but they form only a subspace of dimension $k-1$. Some linear combinations of the $v_{i}$,

$$
v_{1}+\delta v_{2}+\sum_{3 \leq j \leq k-1} \epsilon_{j} v_{j}
$$


will be quadratic forms in the $y_{i}$ of rank 2 . A computation shows that the only linear combinations of the products $u_{i} u_{i+1}$ of rank 2 are of the form

$$
\alpha_{i} u_{i-1} u_{i}+\beta_{i} u_{i} u_{i+1}=u_{i}\left(\alpha_{i} u_{i-1}+\beta_{i} u_{i+1}\right),
$$

for any values of $\alpha_{i}, \beta_{i}, i$. Because the $v_{j}$ span a subspace of codimension 1 , and because we are further restricting to one lower dimension by the choice of the multiplier 1 for $v_{1}$ in the linear combination, we find that for each $i$ there will be one pair $\left(\alpha_{i}, \beta_{i}\right)$ and one set of coefficients $\left(\delta, \epsilon_{j}\right)$ such that

$$
\alpha_{i} u_{i-1} u_{i}+\beta_{i} u_{i} u_{i+1}=u_{i}\left(\alpha_{i} u_{i-1}+\beta_{i} u_{i+1}\right)=v_{1}+\delta v_{2}+\sum_{3 \leq j \leq k-1} \epsilon_{j} v_{j} .
$$

The condition of being rank 2 is an algebraic condition: setting

$$
v_{1}+\delta v_{2}+\sum_{3 \leq j \leq k-1} \epsilon_{j} v_{j}=\sum_{i j} \tau_{i j} y_{i} y_{j},
$$

with $\tau_{i j}=\tau_{j i}$, we find that each $3 \times 3$ submatrix of the $\left(\tau_{i j}\right)$ has vanishing determinant. Each of these determinants is a polynomial equation in $\delta, \epsilon_{j}$. Use resultants to eliminate $\epsilon_{j}$ from this family of polynomial equations (in the ring $\mathrm{Z} / N$ ) and find a single polynomial $F$ of degree $k$ satisfied by $\delta$. We also find $\epsilon_{j}$ as polynomials in $\delta$, by returning to the original equations and eliminating the variables $\epsilon_{i}, i \neq j$.

Thus each solution $\delta$ to $F(\delta)=0$ gives rise to a linear combination of $v_{j}$ which is of rank 2. The root $\delta$ corresponds to that index $i$ for which

$$
v_{1}+\delta v_{2}+\sum_{3 \leq j \leq k-1} \epsilon_{j} v_{j}=u_{i}\left(\alpha_{i} u_{i-1}+\beta_{i} u_{i+1}\right)
$$

We will indicate this correspondence by writing $\delta=\delta_{i}$.

For each solution $\delta=\delta_{i}$, the rows of the resulting matrix $\left(\tau_{i j}\right)$ span a subspace $Y\left(\delta_{i}\right)=Y_{i}$ of $\mathbf{Z}_{p}^{k}$ of rank 2; namely, $Y_{i}$ is spanned by $u_{i}$ and $\alpha_{i} u_{i-1}+\beta_{i} u_{i+1}$.

Observe that $u_{i}, u_{i+2}$, and $\left(\alpha_{i+1} u_{i}+\beta_{i+1} u_{i+2}\right)$ are linearly related, as are $u_{i}, u_{i-2}$, and $\left(\alpha_{i-1} u_{i-2}+\beta_{i-1} u_{i}\right)$. So

$$
u_{i} \in Y_{i} \cap\left(Y_{i+1}+Y_{i+2}\right) \cap\left(Y_{i-1}+Y_{i-2}\right)
$$

This is an algebraic relation among $\delta_{i-2}, \delta_{i-1}, \delta_{i}, \delta_{i+1}$, and $\delta_{i+2}$.

We formulate the relation as the vanishing of several determinants, and reduce the resulting ideal by factoring out any occurrences of $\left(\delta_{i}-\delta_{j}\right), i \neq j$ to assure that $\delta_{i}, \delta_{j}$ are really two different solutions. That is, we consider the ideal formed by $F\left(\delta_{i}\right),\left(F\left(\delta_{i}\right)-F\left(\delta_{j}\right)\right) /\left(\delta_{i}-\delta_{j}\right)$, etc., and the various determinants. We apply the Groebner basis and the Euclidean algorithm to this ideal to find a basis.

Only multiples of some $u_{i}$ satisfy such a relation (19) over $\mathbf{Z} / p$, namely, two different linear relations. We fix a multiple of each $u_{i}$ by normalizing $u_{i}$ to have first coordinate 1 . The linear relations serve to define $u_{i}$ in terms of $\delta_{i}$. 
By similar argument, there is a quadratic equation expressing $\delta_{i+1}$ in terms of $\delta_{i}$, whose two solutions are $\delta_{i+1}$ and $\delta_{i-1}$. The algebraic condition is that the corresponding spaces $Y_{i}, Y_{i+1}$ are in two different triples of subspaces enjoying linear relations:

$$
\operatorname{rank}\left(Y_{i}+Y_{i+1}+Y_{i+2}\right)=\operatorname{rank}\left(Y_{i}+Y_{i+1}+Y_{i-1}\right)=5
$$

We represent the solution of the quadratic equation by $\tau$, and say that $(\delta, \tau)$ generates a pair of 'adjacent' elements $\left(u_{i}, u_{i+1}\right)$ (elements which are multiplied together in the original signature). We think of $\delta$ as generating an extension of degree $k$ over $\mathbf{Z} / N$, and $\tau$ as generating an extension of degree 2 over $\mathbf{Z} / N[\delta] / F(\delta)$. The ability to distinguish the unordered pairs of 'adjacent' roots $\left\{\delta_{i}, \delta_{i+1}\right\}$ makes the system similar, in spirit, to a Galois extension of $\mathbf{Q}$ whose Galois group is the dihedral group on $k$ elements. We will call on this analogy later. (Remark: it is only an analogy, because $\delta$ and $\tau$ really are elements of the ground fields.)

We can get the missing $k$ th equation

$$
v_{k}^{\prime}=\sum_{i} u_{i} u_{i+1} .
$$

The coefficients of $v_{k}^{\prime}$ in terms of $y_{j} y_{\ell}$ ostensibly depend on $\delta_{i}$ and on the pairings $\left(\delta_{i}, \delta_{i+1}\right)$, or equivalently on $(\delta, \tau)$. But the coefficients would come out the same no matter which solution $(\delta, \tau)$ were chosen, that is, no matter whether we assigned the ordering $(1,2,3, \ldots, k)$ or $(3,2,1, k, k-1, \ldots, 4)$ to the solutions $u_{i}$. This means that the coefficients will be in fact independent of $(\delta, \tau)$. They will be expressible in terms of only the coefficients of the original $v_{i}, 1 \leq i \leq k$. This is because they are symmetric (up to dihedral symmetry) in the solutions $\delta_{i}$.

The arguments here are analogous to those of Galois theory. Each coefficient $c$ of $v_{k}^{\prime}$ is expressed as

$$
c=\sum_{0 \leq i<k, 0 \leq j \leq 1} w_{i j} \delta^{i} \tau^{j}
$$

For each of $2 k$ different choices of $(\delta, \tau)$ the value of $c$ comes out the same. Treating (22) as $2 k$ linear equations in the $2 k$ unknowns $w_{i j}$, with coefficients given by $\delta^{i} \tau^{j}$ for various choices of $(\delta, \tau)$, we must find (if the matrix has full rank) that $w_{00}=c$, and $w_{i j}=0$ for $(i, j) \neq(0,0)$.

Now we wish to solve a particular signature. We are given the values $v_{1}, \ldots, v_{k-1}$, and we assign an arbitrary value to $v_{k}^{\prime}$. We have the equations relating $v_{i}$ to $u_{j} u_{j+1}$ :

$$
v_{i}=\sum_{j} b_{i j}^{\prime} u_{j} u_{j+1}
$$

where $b_{i j}^{\prime}$ depends on $\delta_{j}$. Select (symbolically) one pair $(\delta, \tau)$ to fix the first two solutions $\left(u_{1}, u_{2}\right)$, and compute the others in terms of $(\delta, \tau)$. Then we have $b_{i j}^{\prime} u_{j} u_{j+1}$ depending only on $(\delta, \tau)$.

Invert this matrix $b^{\prime}$ to solve for $u_{j} u_{j+1}$ in terms of the given $v_{i}$ and $(\delta, \tau)$. Now assign

$$
u_{1}=\xi \text {, }
$$


where $\xi$ is an unknown. Compute

$u_{2}=\frac{\left(u_{1} u_{2}\right)}{\xi}, u_{3}=\frac{\xi\left(u_{2} u_{3}\right)}{\left(u_{1} u_{2}\right)}, u_{4}=\frac{\left(u_{1} u_{2}\right)\left(u_{3} u_{4}\right)}{\xi\left(u_{2} u_{3}\right)}, \ldots, u_{1}=\frac{\left(u_{1} u_{2}\right)\left(u_{3} u_{4}\right) \ldots\left(u_{k} u_{1}\right)}{\xi\left(u_{2} u_{3}\right) \ldots\left(u_{k-1} u_{k}\right)}$

The last equation gives a quadratic equation which $\xi$ must satisfy:

$$
\left(u_{1} u_{2}\right)\left(u_{3} u_{4}\right) \ldots\left(u_{k} u_{1}\right)=\xi^{2}\left(u_{2} u_{3}\right) \ldots\left(u_{k-1} u_{k}\right)
$$

We do not solve for $\xi$ (we cannot). So now we have three algebraic unknowns: $\delta, r, \xi$, of successive degrees $k, 2,2$.

These equations give $u_{i}$ in terms of $\delta, \tau, \xi$. Notice that each $u_{i}$ is an odd function of $\xi$ : either $\xi$ times a function of $(\delta, \tau)$ or $\xi^{-1}$ times a function of $(\delta, \tau)$. We also have $u_{i}$ as linear combinations of $y_{j}$ with coefficients depending on $(\delta, \tau)$. Solve for $y_{j}$ in terms of $(\delta, \tau, \xi)$, and note that $y_{j}$ is again an odd function of $\xi$.

Now each product $y_{j} y_{\ell}$ will be a function only on $(\delta, \tau)$, since it will be an even function of $\xi$, and we know $\xi^{2}$ in terms of $(\delta, \tau)$. But again the value $y_{j} y_{\ell}$ will be independent of the dihedral ordering $(1,2,3, \ldots, k)$ versus $(3,2,1, k, k-1, \ldots, 4)$, and thus independent of the choice of solutions $(\delta, \tau)$. That means, by standard Galois theory arguments, that $(\delta, \tau)$ will not appear in the expressions of $y_{j} y_{\ell}$.

So we have found the products $y_{j} y_{\ell}$ in terms of the given coefficients, the given values $v_{1}, v_{2} \ldots, v_{k-1}$, and the assumed value $v_{k}^{\prime}$. We have given a valid signature.

\section{Comments and extensions}

\subsection{Working $\bmod N$ versus working $\bmod p$}

Some justification is needed to go from calculations $\bmod p$ to calculations $\bmod$ $N$. In section 1, we basically use tools from linear algebra such as Gaussian elimination or determinants. Thus all computations go through regardless the fact that $N$ is composite. The situation is a bit more subtle in section 2 . For instance, $F$ has $k$ solutions $\bmod p$ but $k^{2}$ solutions $\bmod N$, each obtained by mixing some solution $\bmod p$, with some solution $\bmod q$. But if we consider only the image, $\bmod p$, of our calculations $\bmod N$, things are all right: the symmetric functions of the $k$ roots of a polynomial are expressible in terms of the coefficients of the polynomial, and the expressions of the products $y_{j} y_{\ell}$ in terms of the coefficients of the public key are valid $\bmod p$. They are also valid $\bmod q$, and the Chinese remainder theorem suffices to make them valid $\bmod N$. This in spite of the fact that a solution $\delta$ of $F \bmod N$ might well mix different solutions $\delta_{i} \bmod p$ and $\delta_{j} \bmod q$. Since we never explicitly solve for $\delta$, but only work with it symbolically and use the fact that $F(\delta)=0 \bmod N$, we never are in danger of factoring $N$. 


\subsection{Extension to the case $s>1$ (Sketch)}

The case $s>1$ is more complicated. Suppose again that we have $k$ variables $y_{1}, y_{2}, \ldots, y_{k}$, with $k$ odd, whose pairwise products constitute the signature, and that the hashed message has $k-s$ quantities $v_{1}, v_{2}, \ldots, v_{k-s}$, together with coefficients $c_{i j \ell}$ expressing $v_{i}$ in terms of $y_{j} y_{\ell}$. Suppose for simplicity that $s>1$ is odd, so that $k-s$ is even.

Some linear combinations of the $k-s$ quadratic forms $v_{i}$ will have rank $s+1$. Namely, for each index set $I \subseteq\{1,2, \ldots, k\}$ of size $(s+1) / 2$ such that $\forall i, j \in I$ : $|i-j| \geq 2$, there is such a linear combination of the form

$$
\sum_{i \in I} u_{i}\left(\alpha_{i I} u_{i-1}+\beta_{i I} u_{i+1}\right)
$$

The number of such index sets $I$ is

$$
\frac{k}{\frac{s+1}{2}}\left(\begin{array}{c}
k-\frac{s+3}{2} \\
\frac{s-1}{2}
\end{array}\right)
$$

There are more than $k$ linear combinations, leading to increased complication. The space $Y_{I}$, spanned by rows of the corresponding quadratic form, contains $u_{i}$ for each index $i \in I$. So each $u_{i}$ is in the intersection of a large number of subspaces $Y_{I}$, and hopefully only multiples of $u_{i}$ will be in such an intersection. This algebraic condition should distinguish the $u_{i}$, hopefully indexing them by the roots $\delta$ of some polynomial $F(\delta)$ of degree $k$. Pairs $\left\{u_{i}, u_{i+2}\right\}$ of solutions with index differing by 2 should be distinguished by appearing together in many different subspaces $Y_{I}$. Using this we would be able to distinguish pairs $\left\{u_{i}, u_{i+1}\right\}$. We would fabricate the missing equations: for $j=k-s+1, \ldots, k$, let $u_{i(j)}^{\prime}$ be a multiple of $u_{i}$, normalized to have a 1 in position $j$, and set $v_{j}^{\prime}=\sum_{i} u_{i(j)}^{\prime} u_{i+1(j)}^{\prime}$.

\subsection{The case $k=3, s=1$}

In the special case $k=3, s=1$, where we must satisfy two quadratic equations in three variables, we can employ an ad hoc method, since the methods outlined above don't work. Take a linear transformation of the two quadratic equations so that the right-hand side of one equation vanishes; that is, if the given values are $v_{1}$ and $v_{2}$, take $v_{2}$ times the first equation minus $v_{1}$ times the second. This gives a homogeneous quadratic equation in three variables $y_{1}, y_{2}, y_{3}$ :

$$
\sum_{i j} c_{i j} y_{i} y_{j}=0
$$

The second equation is inhomogeneous:

$$
\sum_{i j} d_{i j} y_{i} y_{j}=d_{0}
$$


By setting $z_{1}=y_{1} / y_{3}, z_{2}=y_{2} / y_{3}$ in (29), we obtain an inhomogeneous quadratic equation in two variables $z_{1}, z_{2}$. We can easily find an affine change of basis from $z_{1}, z_{2}$ to $z_{1}^{\prime}, z_{2}^{\prime}$ which transforms the equation to the form

$$
c_{11}^{\prime} z_{1}^{\prime 2}+c_{12}^{\prime} z_{1}^{\prime} z_{2}^{\prime}+c_{22}^{\prime} z_{2}^{\prime 2}=c_{0}^{\prime} \bmod N
$$

and a further linear change of variables to $z_{1}^{\prime \prime}, z_{2}^{\prime \prime}$ yielding

$$
c_{11}^{\prime \prime} z_{1}^{\prime \prime 2}+c_{22}^{\prime \prime} z_{2}^{\prime 2}=c_{0}^{\prime \prime} \bmod N
$$

which can be solved by the Pollard [2] attack on the Ong-Schnorr-Shamir [1] scheme. We find from this a set of ratios $y_{j} / y_{3}$, and, by extension, a set of ratios $y_{i} y_{j} / y_{3}^{2}$, satisfying (29). Setting $y_{3}^{2}=\lambda$, the second equation (30) becomes a linear equation in $\lambda$. Thus we find a consistent set of pairwise products $y_{i} y_{j}$ satisfying the desired equations (29), (30).

\subsection{Open questions}

The birational permutation signature scheme has many instances, of which we have attacked only the first few examples. For a more complex instance of the scheme, the ideas of the present paper will still apply: the trap door conditions lead to algebraic equations on the coefficients of the transformations, and we hope to gather enough such equations to make it possible to solve them by g.c.d. or Groebner basis methods. But, for any specific instance, it remains to see whether the ideas of the present paper would be sufficient to mount an attack.

One general theme is that when solutions of the algebraic equations enjoy a symmetry, it makes the equations harder to solve, but we don't need to solve them, since the final solution will enjoy the same symmetry, and quantities symmetric in the roots of the equation can be expressed in terms of the coefficients of the equation alone, not in terms of the roots. When the roots fail to enjoy a symmetry, they can be distinguished by algebraic conditions, which yield further algebraic equations, and the Groebner basis methods have more to work with. This gives us hope that the methods outlined in this paper will apply with some generality to many instances of the birational permutation signature scheme.

\section{References}

1. H. Ong, C. P. Schnorr, and A. Shamir: A fast signature scheme based on quadratic equations. Proc. 16th ACM Symp. Theory of Computing, pp.208-216; 1984.

2. J. M. Pollard and C. P. Schnorr: An efficient solution of the congruence $x^{2}+k y^{2}=m$ $(\bmod n)$. IEEE Trans. Inform. Theory vol IT-33 no 5, pp.702-709; Sept., 1987.

3. A. Shamir: Efficient signature schemes based on birational permutations. Manuscript March 1993. To appear, Crypto 99. 